\title{
Conformational studies of blood group A and blood group B oligosaccharides using NMR residual dipolar couplings
}

\author{
Hugo F. Azurmendi, ${ }^{\dagger}$ C. Allen Bush* \\ Department of Chemistry and Biochemistry, University of Maryland-Baltimore County, 1000 Hilltop Circle, Baltimore, MD 21250, USA
}

Received 11 January 2002; accepted 4 March 2002

\begin{abstract}
The conformations of two synthetic trisaccharides of blood group A and B ( $\alpha$-L-Fuc $p-(1 \rightarrow 2)-[\alpha-\mathrm{D}-\mathrm{Gal} p$ NAc- $(1 \rightarrow 3)]-\alpha-\mathrm{D}-\mathrm{Gal} p$ and $\alpha$-L-Fuc $p-(1 \rightarrow 2)$-[ $\alpha$-D-Gal $p-(1 \rightarrow 3)]-\alpha$-D-Gal $p$, respectively) and of a type A tetrasaccharide alditol, Fuc $p-(1 \rightarrow 2)-[\alpha-\mathrm{D}-\mathrm{Gal} p-$ NAc- $(1 \rightarrow 3)$ ]- $\beta$-D-Gal $p-(1 \rightarrow 3)-G a 1 N A c-o l$, were studied by NMR measurements of one-bond C-H residual dipolar couplings in partially oriented liquid crystal solutions. The conformations of the three oligosaccharides were analyzed by generating thousands of structures using a Monte-Carlo method. Two different strategies were applied to calculate theoretical dipolar couplings for these structures. In the first method, the orientation of the molecule was calculated from the optimal fit of the molecular model to the experimental data, while in the second method the orientation tensor was calculated directly from the moment of inertia of the molecular model. Both methods of analysis give similar results but with slightly better agreement with experiment for the former one. The analysis of the results implies a single unique conformation for both blood group epitopes in solution in disagreement with theoretical models suggesting the existence of two conformers in solution. (C) 2002 Elsevier Science Ltd. All rights reserved.
\end{abstract}

Keywords: Blood group; NMR, residual dipolar coupling; Oligosaccharide conformation

\section{Introduction}

The blood group A and B determinants are two of the most important antigens present on erythrocyte membranes of apes and man, playing a crucial roll in blood transfusion and tissue transplantation. The determinant $\mathrm{A}$ is the trisaccharide $\alpha$-L-Fuc $p-(1 \rightarrow 2)-[\alpha-\mathrm{D}-$ Gal $p$ NAc- $(1 \rightarrow 3)]-\mathrm{D}-\mathrm{Gal} p$, which differs from $\mathrm{B}$ in that the latter lacks the NAc group. Earlier studies of these molecules using NOE and simple molecular modeling have concluded that they are essentially rigid, ${ }^{1-4}$ but later studies by more sophisticated molecular modeling suggested the possibility of two conformers in solution. ${ }^{5}$ New experimental studies of blood group A based on NMR relaxation measurements reported agreement

\footnotetext{
* Corresponding author:. Tel.: + 1-410-4552506; fax: + 1410-4552608.

E-mail address: bush@umbc.edu (C.A. Bush).

† Permanent address: Grupo de Biofísica, Departamento de Física, Universidad Nacional del Sur, Bahía Blanca, Argentina.
}

with the two conformer model, ${ }^{6}$ but later reports on blood group B using NMR long-range coupling constants concluded the presence of just one conformer. ${ }^{7}$

Residual dipolar couplings (RDC) measured in oriented media ${ }^{8}$ provide a relatively new technique that is having a high impact in structure determinations of proteins and oligosaccharides. In general they are easy to measure with high accuracy, and they give important structural and dynamic information. Until recently, most structural characterizations of oligosaccharides in solution have been based on NMR NOEs, non-selective $\mathrm{T}_{1}$ and scalar couplings, the interpretation of which may be imprecise because of the small number of cross peaks in NOE, difficulties in the determination of longrange scalar couplings, and inaccuracies in the interpretation of $\mathrm{T}_{1}$ values. $^{9-12}$ These techniques usually have been complemented with computational methods such as molecular dynamics, which have provided a deeper understanding of the structural and dynamic properties of these systems. ${ }^{13}$ The development of the RDC method has provided a new tool for obtaining longer range structural information, and it is being widely 
applied to study carbohydrates. ${ }^{14-20}$ This method is particularly well suited for the study of molecules with internal motions of the first kind having motions on a pico-second time scale within a local minimum with limited excursions of the glycosidic dihedral angles $\left( \pm 15^{\circ}\right),{ }^{21}$ although extensions for the analysis of flexible models of oligosaccharides have been recently proposed. ${ }^{22,23}$

The aim of this work is to determine whether the solution conformations of blood group A and B oligosaccharides are adequately described by a relatively rigid model with internal motion of the first kind or whether a more flexible model with exchange between conformers is required. One-bond $\mathrm{C}-\mathrm{H}$ residual dipolar couplings in partially oriented liquid crystal solutions were analyzed by energy calculations and dipolar coupling simulations. Two different strategies were applied to calculate theoretical dipolar couplings for these structures, one based on orienting the model to get the best fit with the experimental values, and the other by TRacking Alignment from the Moments of Inertia Tensor (TRAMITE) of the models. ${ }^{24}$

\section{Experimental}

Sample preparation. - A $\sim 15 \%$ bicelle stock solution in molar ratio 3:1 of 1,2-di- $O$-tridecanyl-sn-glycero-3phosphocholine (13:0 Diether PC) and 1,2-di- $O$-hexylsn-glycero-3-phosphocholine (6:0 Diether $\mathrm{PC}$ ) in $\mathrm{D}_{2} \mathrm{O}$ was prepared. Lipids were purchased from Avanti Polar Lipids, Inc. This bicelle media was chosen because its improved chemical stability with respect to conventional phospholipid bicelles. The solution was mixed with vortexing in several cycles of heating to $40{ }^{\circ} \mathrm{C}$ and cooling on ice.

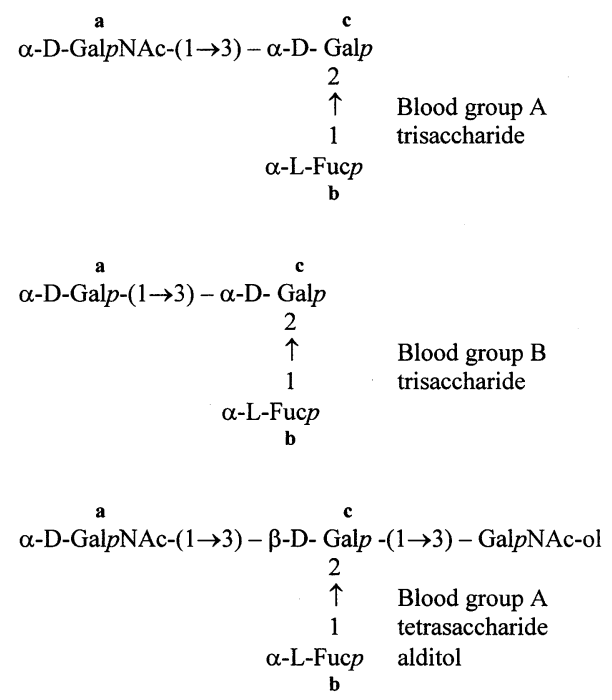

Scheme 1. Representation of the blood groups A and B trisaccharide and the blood group A tetrasaccharide alditol.
Blood group A trisaccharide and blood group B trisaccharide were purchased from Calbiochem-Novabiochem Corporation. Blood group A tetrasaccharide alditol was isolated by alkaline borohydride degradation of an A-active pig mucin and was provided by $\mathrm{Dr}$ Thomas Gerken of Case-Western Reserve University. Each oligosaccharide was exchanged in ${ }^{2} \mathrm{H}_{2} \mathrm{O}$ and lyophilized for three cycles. A sample of $\sim 10 \mathrm{mg}$ of each one was dissolved in $0.25 \mathrm{~mL}$ of $\mathrm{D}_{2} \mathrm{O}$, and 0.25 $\mathrm{mL}$ of bicelle stock solution was added to obtain a final bicelle solution of $\sim 7.5 \%$. Each sample was homogenized by vortexing in several cycles of heating to $40^{\circ} \mathrm{C}$ and cooling on ice. Scheme 1 represents the three compounds.

NMR spectroscopy. - NMR data were acquired on a GE-Omega PSG 500 NMR instrument and processed on a Silicon Graphics workstation using FELIX 2.3 software (Biosym Technologies, San Diego, CA). Residual dipolar coupling values, ${ }^{1} D_{\mathrm{CH}}$, were measured for each sample of oligosaccharide dissolved in the bicelles from the difference in the splitting of two $t_{1}$-coupled, gradient HSQC spectra ( $t_{1}$-coupled gHSQC), ${ }^{25}$ acquired at 35 and $19^{\circ} \mathrm{C}$, corresponding to the oriented and isotropic solution, respectively. To maximize the resolution in the indirect dimension, three sets of $t_{1}$-folded experiments were acquired with spectral widths of $4000 \mathrm{~Hz}$ in the indirect dimension, one with ${ }^{13} \mathrm{C}$ carrier position in the center of the anomeric region ( $\sim 95 \mathrm{ppm}$ ), another in the center of the ring region $(\sim 70 \mathrm{ppm})$, and the last one in the methyl region $(\sim 20 \mathrm{ppm})$. For each experiment, a $2 \mathrm{D}$ matrix of $1024 \times 256$ complex FID data points was acquired. The 2D FIDs were apodized in both dimensions with a $90^{\circ}$-shifted sine-bell function and zero-filling to give, after Fourier transformation, a 2D spectrum of $1024 \times$ 1024 real points. The column corresponding to each $\mathrm{C}-\mathrm{H}$ signal was carefully phased and stored. After an inverse Hilbert transformation and a zero-filling to 16 $\mathrm{K}$, the retransformed vector showed a digital resolution of $0.2 \mathrm{~Hz}$ /point. Vectors from the $35{ }^{\circ} \mathrm{C}$ spectrum were compared with the corresponding vector from the $19^{\circ} \mathrm{C}$ spectrum, and the offset required for superposition of the multiplet components was used to calculate the sign and magnitude of ${ }^{1} D_{\mathrm{CH}}$. In the case of methyl groups, the difference in splitting in the outer quartet components was used. ${ }^{26}$ The estimated experimental error with this procedure for these samples was $\pm 0.5 \mathrm{~Hz}$.

Molecular modeling and computation.-Molecular modeling was used as a tool for analysis, principally as a source of model structures to interpret dipolar couplings as is explained below. The model structures were generated using MACROMODEL $5.0^{27}$ in 10,000 cycles of Monte-Carlo (MC) steps with the MM3 force-field. ${ }^{28}$ Better quality structures were obtained using the GB/ SA continuum solvent model ${ }^{29}$ implemented in MACROMODEL mimicking water dielectric values. This protocol 
has been reported as a suitable one in reproducing experimental coupling constants for sialyllactones, ${ }^{30}$ and more generally for sampling carbohydrate conformations. ${ }^{31}$

The general theoretical expression accounting for dipolar couplings is: $:^{8,32}$

${ }^{1} D^{\mathrm{CH}}(\theta, \phi)=D_{\mathrm{a}}^{\mathrm{CH}}\left[\left(3 \cos ^{2} \theta-1\right)+\frac{3}{2} R \sin ^{2} \theta \cos 2 \phi\right]$

with

$R=D_{\mathrm{r}}^{\mathrm{CH}} / D_{\mathrm{a}}^{\mathrm{CH}}$

$D_{\mathrm{a}}^{\mathrm{CH}}=\frac{1}{3}\left[D_{z z}^{\mathrm{CH}}-\left(D_{x x}^{\mathrm{CH}}+D_{y y}^{\mathrm{CH}}\right) / 2\right]$

$D_{\mathrm{r}}^{\mathrm{CH}}=\frac{1}{3}\left[D_{x x}^{\mathrm{CH}}-D_{y y}^{\mathrm{CH}}\right]$

$D_{\mathrm{a}}^{\mathrm{CH}}$ and $D_{\mathrm{r}}^{\mathrm{CH}}$ are the axial and rhombic components in units of hertz of the traceless second rank diagonal tensor $\mathbf{D}$, with $\left|D_{z z}^{\mathrm{CH}}\right|>\left|D_{y y}^{\mathrm{CH}}\right| \geq\left|D_{x x}^{\mathrm{CH}}\right|, R$ is called the rhombicity (which ranges from 0 to $2 / 3$ ), and $\theta$ and $\phi$ are the spherical polar coordinates describing the orientation of the $\mathrm{C}-\mathrm{H}$ vector with respect to the $z$ axis of the system. For the case of liquid crystal bicelle medium, it can be proved that $D_{\mathrm{a}}^{\mathrm{CH}}=-\left(\mu_{0} h\right)$ $16 \pi) S \gamma_{\mathrm{C}} \gamma_{\mathrm{H}}\left\langle r_{\mathrm{CH}}^{-3}\right\rangle A_{\mathrm{a}},{ }^{33}$ where $A_{\mathrm{a}}$ is the unitless axial component of the molecular alignment tensor $\mathbf{A}, S$ is the generalized order parameter for internal motions of the $\mathrm{C}-\mathrm{H}$ pair, ${ }^{34} \mu_{0}$ is the magnetic permeability of vacuum, $\gamma_{\mathrm{C}}$ and $\gamma_{\mathrm{H}}$ are the respective gyromagnetic ratios for $\mathrm{C}$ and $\mathrm{H}, h$ is the Planck's constant and $r_{\mathrm{CH}}$ is the distance between $\mathrm{C}$ and $\mathrm{H}$ with \langle\rangle brackets indicating vibrational average.

Two methods were used to interpret the experimental dipolar coupling values. In both, the model structures obtained by molecular modeling are used to determine the orientation tensor. In the first one each model is iteratively oriented in the best way to minimize the merit function $\chi$ defined by:

$\chi=\left[\frac{1}{n} \sum_{1}^{n}\left({ }^{1} D_{\mathrm{CH}}^{\mathrm{exp}}-{ }^{1} D_{\mathrm{CH}}^{\mathrm{calc}}\right)^{2}\right]^{1 / 2}$

where $n$ is the number of $\mathrm{C}-\mathrm{H}$ dipolar vectors. RDC from methyl groups can be included in the calculations taking into account that, as shown previously, ${ }^{26}$ rapid rotation of tetrahedral methyl groups scales the ${ }^{13} \mathrm{C}-{ }^{1} \mathrm{H}$ dipolar couplings by $-1 / 3$, due to rapid rotation. Thus, the threefold larger change in splitting observed for the outer methyl quartet components is the same magnitude but opposite in sign to that for a $\mathrm{C}-\mathrm{H}$ bond oriented in the same direction as the C-5-C-6 bond in the 6-deoxy sugar. Scaling by the ratio of bond lengths, $\sim r_{\mathrm{CH}} / r_{\mathrm{CC}}$, allows the experimental values for the methyl groups in the 6-deoxy sugars to be introduced directly in the calculation using the bond vector $\mathrm{C}-\mathrm{CH}_{3}$ as a normal $\mathrm{C}-\mathrm{H}$ vector. In the first method for analysis, the orientation tensor that minimizes $\chi$ in Eq. (2) for a given structure model was found using a previously described algorithm. ${ }^{20}$ In the systematic search for a given fixed molecular model, the Euler angles describing the orientation tensor are cycled through eight values each for three values of $R(0,1 / 3,2 / 3)$. The initial $D_{\mathrm{a}}$ is estimated from the maximum experimental RDC value as $D_{\mathrm{CH}}^{\mathrm{MAX}} / 2 .{ }^{32,34}$ The algorithm discards bad orientations rapidly focusing on the better ones using Powell optimization to obtain the lowest $\chi$ value possible for that model structure. Experimental error is simulated by introducing variations in ${ }^{1} D_{\mathrm{CH}}^{\mathrm{exp}}$, randomly selected from a Gaussian distribution whose width is adjusted through an input parameter for each iteration of the algorithm.

Unlike the first method, the second one assumes that the molecular shape determines the orientation ignoring electrostatic effects on the orientation of the solute. ${ }^{35}$ In this case the orienting media used were neutral bicelles with neutral sugars consistent with this assumption. The method, named tracking alignment from the moment of inertia tensor (TRAMITE) has been described and is briefly summarized here. ${ }^{24}$ It assumes that the eigenvalues of the moment of inertia tensor $\mathbf{I}$ are parallel to those of the orientation tensor $\mathbf{A}$, and that the eigenvalues of $\mathbf{A}$ are proportional to the asymmetries of the solute in the planes defined by pairs of eigenvectors of I, without any explicit correspondence between the axes of $\mathbf{I}$ and $\mathbf{A}$. This leads to the definition:

$A_{i i}^{\prime}=I_{i i}-\frac{I_{j j}+I_{k k}}{2} \quad(i, j, k=1,2,3)$

$A_{i i}^{\prime}$ are the elements of a trial matrix that arranged from greater to lesser absolute values build up a tensor with the same properties of the orientation tensor. By sorting the eigenvalues of $\mathbf{I}$ as $I_{33}>I_{22}>I_{11}$, two possible situations arise defining the correspondence between the principal axes of $\mathbf{A}$ and $\mathbf{I}$ :

$$
\begin{aligned}
& A_{33}^{\prime}+A_{11}^{\prime}=-A_{22}^{\prime}>0 \Rightarrow A_{z z}=A_{33}^{\prime}, \quad A_{y y}=A_{11}^{\prime}, \\
& A_{x x}=A_{22}^{\prime} \\
& A_{33}^{\prime}+A_{11}^{\prime}=-A_{22}^{\prime}<0 \Rightarrow A_{z z}=A_{11}^{\prime}, \quad A_{y y}=A_{33}^{\prime}, \\
& A_{x x}=A_{22}^{\prime}
\end{aligned}
$$

With this definition, the correspondence among the axes is obtained, and it is possible to calculate the rhombicity by means of the relations (2). In this way, dipolar couplings can be predicted through Eq. (1) except for a scale factor dependent on experimental conditions, which can be obtained by fitting the simulated ${ }^{1} D$ values to minimize the merit function $\chi$ (Eq. (3)). The algorithm was implemented on MATHEMATICA 4.1 software from Wolfram Research, Inc. The use of the eigenvectors of the moment of inertia tensor to 
Table 1

Chemical shifts of the blood groups A (trisaccharide and tetrasaccharide alditol) and blood group B

\begin{tabular}{|c|c|c|c|c|c|c|}
\hline \multirow[t]{2}{*}{ Atom } & \multicolumn{2}{|c|}{ Blood group A } & \multicolumn{2}{|c|}{ Blood group A-alditol } & \multicolumn{2}{|c|}{ Blood group B } \\
\hline & ${ }^{1} \mathrm{H}$ & ${ }^{13} \mathrm{C}$ & ${ }^{1} \mathrm{H}$ & ${ }^{13} \mathrm{C}$ & ${ }^{1} \mathrm{H}$ & ${ }^{13} \mathrm{C}$ \\
\hline $1 \mathrm{a}$ & 5.137 & 93.15 & 5.192 & 92.56 & 5.216 & 94.70 \\
\hline $2 \mathrm{a}$ & 4.225 & 50.93 & 4.249 & 50.32 & 3.869 & 69.48 \\
\hline $3 a$ & 3.976 & 68.95 & 3.924 & 68.74 & 3.944 & 70.66 \\
\hline $4 a$ & 3.978 & 69.99 & 4.022 & 69.32 & 3.961 & 70.72 \\
\hline $5 \mathrm{a}$ & 4.405 & 72.13 & 4.151 & 72.23 & 4.389 & 72.10 \\
\hline $6 a$ & 3.768 & 62.75 & 3.782 & 61.17 & 3.744 & 62.53 \\
\hline $\mathrm{CH}_{3}$ (NAc) & 2.044 & 23.31 & 2.057 & 23.02 & & \\
\hline $1 b$ & 5.120 & 101.84 & 5.385 & 99.96 & 5.114 & 102.02 \\
\hline $2 b$ & 3.796 & 69.28 & 3.803 & 69.16 & 3.799 & 69.26 \\
\hline $3 b$ & 3.912 & 70.72 & 3.802 & 70.68 & 3.912 & 70.72 \\
\hline $4 b$ & 3.811 & 73.10 & 3.814 & 72.82 & 3.808 & 73.13 \\
\hline $5 b$ & 4.155 & 68.57 & 4.329 & 68.16 & 4.160 & 68.60 \\
\hline $6 b$ & 1.225 & 16.60 & 1.225 & 16.60 & 1.213 & 16.59 \\
\hline $1 \mathrm{c}$ & 5.369 & 97.07 & 4.693 & 101.55 & 5.368 & 93.19 \\
\hline $2 c$ & 3.972 & 75.13 & 3.897 & 74.56 & 3.992 & 75.40 \\
\hline $3 c$ & 4.134 & 72.04 & 4.012 & 76.31 & 4.160 & 72.68 \\
\hline $4 c$ & 4.244 & 65.69 & 4.220 & 64.08 & 4.311 & 65.96 \\
\hline $5 c$ & 4.066 & 71.41 & 3.714 & 76.12 & 4.104 & 71.51 \\
\hline $6 c$ & 3.727 & 62.50 & 3.768 & 61.94 & 3.757 & 62.53 \\
\hline $1 d$ & & & 3.787 & 61.76 & & \\
\hline $2 \mathrm{~d}$ & & & 4.300 & 53.30 & & \\
\hline $3 d$ & & & 4.097 & 75.34 & & \\
\hline $4 d$ & & & 3.594 & 70.29 & & \\
\hline $5 d$ & & & 4.112 & 70.49 & & \\
\hline $6 \mathrm{~d}$ & & & 3.655 & 63.50 & & \\
\hline
\end{tabular}

a, GalNAc $\alpha(1 \rightarrow 3)$ or Gal $\alpha(1 \rightarrow 3)$; b, Fuc $\alpha(1 \rightarrow 2)$; c, Gal $\alpha$ or Gal $\beta(1 \rightarrow 3)$; d, GalNAc-ol.

predict the principal axes of the orientation tensor has been reported also by Almond et al. ${ }^{36}$

Further analysis and graphs were done with ORIGIN 6.0 (Microcal Software, Inc., Northampton, MA). Glycosidic dihedral angles were defined according to the IUPAC heavy-atom convention in which $\phi$ is defined by $\mathrm{O}_{5}-\mathrm{C}_{1}-\mathrm{O}_{1}-\mathrm{C}_{x}$ and $\psi$ by $\mathrm{C}_{x-1}-\mathrm{C}_{x}-\mathrm{O}_{1}-\mathrm{C}_{1}$.

\section{Results and discussion}

The trisaccharides are present in at least three different forms in solution, corresponding to the occurrence of $\alpha$ and $\beta$ anomers of the reducing galactopyranoside, plus another corresponding to a reducing $\alpha$-galactofuranoside. Traces on a $\beta$-galactofuranoside are also present. In spite of the complex anomeric equilibrium, the reducing $\alpha$-galactopyranosidic residue clearly predominates and was used for this study. The ${ }^{1} \mathrm{H}$ and ${ }^{13} \mathrm{C}$ chemical shift assignment for blood group A trisaccharide has been previously reported. ${ }^{37}$ Most of those assignments were confirmed, but some discrepancies arose in interpreting the RDC measurements. With the original assignments, axial $\mathrm{C}-\mathrm{H}$ vectors within the GalNAc residue were completely different as were some of those in the Gal residue, suggesting that some assignments had been interchanged. This was confirmed through an HMBC experiment. Because of the possibility of other errors, all the assignments were checked with the traditional techniques of COSY, TOCSY, HMQC, and HMBC. The same experiments and analysis were performed on the blood group A tetrasaccharide alditol for which only ${ }^{1} \mathrm{H}$ assignments had been reported by Dua et al. ${ }^{38}$

For the blood group B trisaccharide, complete assignments were available for the $\beta$-anomeric glycoside by Otter et $\mathrm{al}^{7}$ which were used as a guide. The complete results are summarized in Table 1.

When the bicelle medium was introduced, the chemical shifts were very similar to those obtained for the oligosaccharides in $\mathrm{D}_{2} \mathrm{O}$, suggesting that the conformations of the trisaccharides are not affected by the presence of the lipids. One-bond $\mathrm{C}-\mathrm{H}$ residual dipolar couplings were measured in dilute bicelle media at 7.5\% 
concentration composed of (13:0 Diether PC/6:0 Diether PC) 3:1, for all three oligosaccharides. Dipolar couplings were determined from the splittings in the ${ }^{13} \mathrm{C}$ dimension of a $t_{1}$-coupled g-HSQC experiment. ${ }^{25}$ An example of the HSQC spectra, corresponding to the isotropic and oriented blood group A trisaccharide in the anomeric region, is given in Fig. 1. The experimen- tal dipolar couplings obtained for these compounds are given in Table 2 and plotted in Fig. 2.

Several thousand structure models were generated for each oligosaccharide using the program MACROMODEL ${ }^{27}$ with the aim of sampling the greatest amount of the glycosidic angular space accessible to the molecules without undue bias to the possible structures

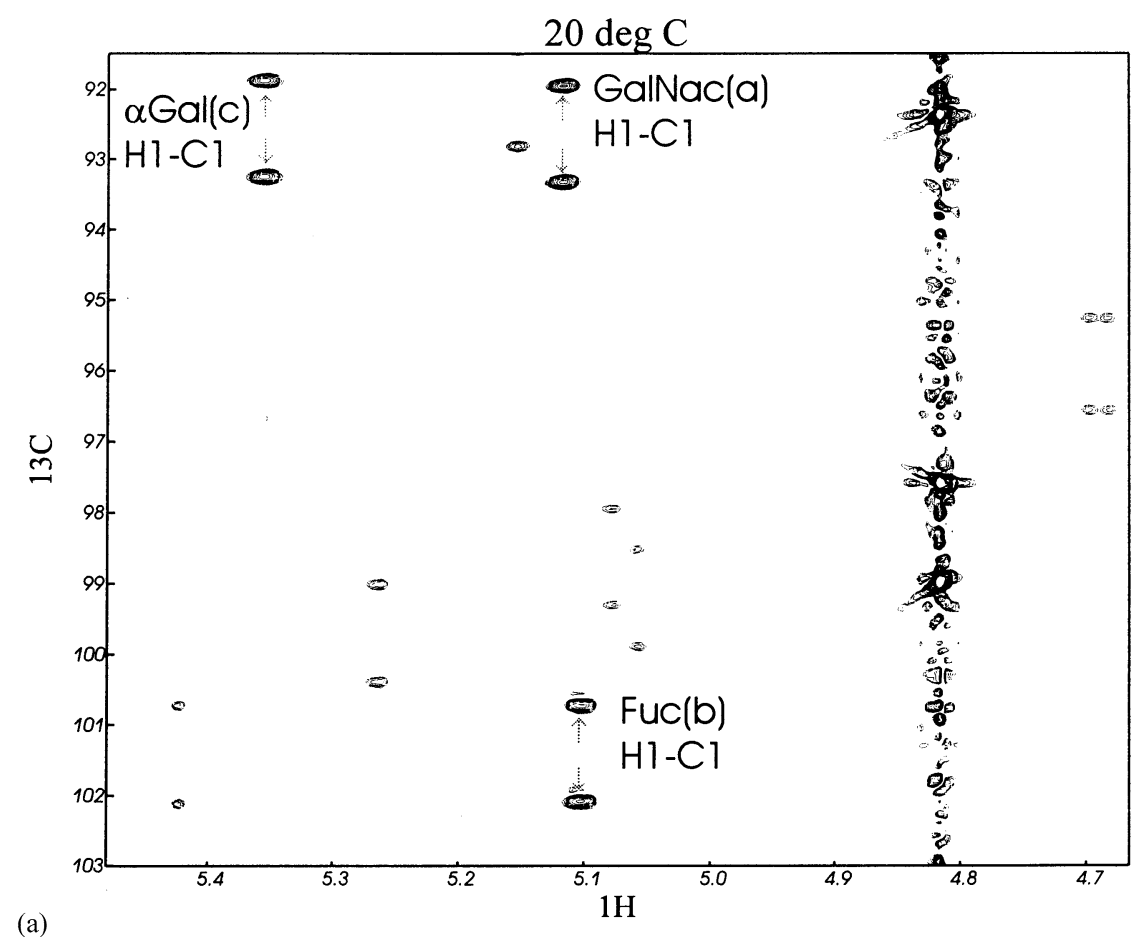

(a)

35 deg. C

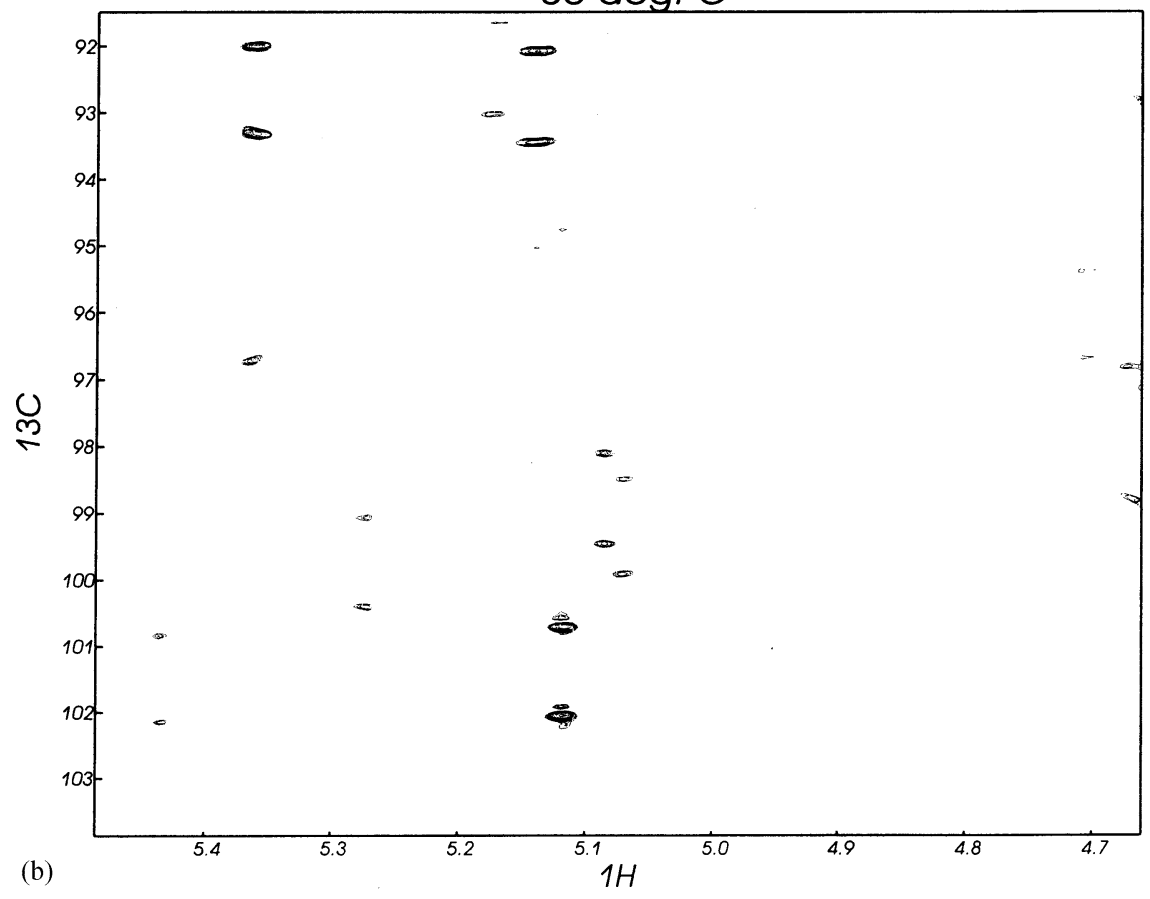

Fig. 1. (a) ${ }^{13} \mathrm{C}$-coupled HSQC spectrum of the anomeric region of the blood group A trisaccharide at $20{ }^{\circ} \mathrm{C}$ (isotropic) and (b) at $35^{\circ} \mathrm{C}$ (oriented). Peaks corresponding to the major anomer are marked. Smaller peaks, not seen at higher contour level, arise from other anomers of the reducing terminal galactose residue, and were not used in the analysis. 
Table 2

Experimental residual dipolar couplings in $\mathrm{Hz}$ for the blood groups A (BGA) and B (BGB) trisaccharide and blood group A tetrasaccharide alditol BGA-ol

\begin{tabular}{lrrr}
\hline Atom pairs & BGA & BGB & BGA-ol \\
\hline H-1-C-1a & -0.6 & -1.8 & -7.4 \\
H-2-C-2a & -7.0 & -5.5 & 0.5 \\
$\mathrm{CH}_{3}$ (NAc) & -2.0 & & \\
H-3-C-3a & -6.0 & -7.0 & 2.9 \\
H-4-C-4a & 0.0 & -1.0 & -7.0 \\
H-5-C-5a & -5.0 & -6.6 & 0.9 \\
H-1-C-1b & -2.0 & 6.4 & 4.6 \\
H-2-C-2b & 5.0 & 2.0 & 4.4 \\
H-3-C-3b & 3.0 & 0.6 & 5.1 \\
H-4-C-4b & -1.2 & 4.0 & -1.9 \\
H-5-C-5b & 5.0 & -0.1 & 5.1 \\
CH & 0.0 & 4.0 & \\
H-1-C-1c & -7.5 & -6.5 & 5.5 \\
H-2-C-2c & 4.0 & 7.5 & -10.3 \\
H-3-C-3c & 5.0 & 7.5 & -8.5 \\
H-4-C-4c & -6.0 & -7.4 & 6.3 \\
H-5-C-5c & 4.0 & 6.5 & -7.2 \\
\hline
\end{tabular}

a, $\operatorname{Gal} p \mathrm{NAc} \alpha(1 \rightarrow 3)$ or $\operatorname{Gal} p \alpha(1 \rightarrow 3)$; b, Fucp $\alpha(1 \rightarrow 2)$; c, $\mathrm{Gal} p, \alpha$ or $\mathrm{Gal} p \beta(1 \rightarrow 3)$. by the molecular modeling. To achieve this goal, two different Monte-Carlo protocols were used, both using the MM3 force field. ${ }^{28}$ In the first method, all exocyclic dihedral angles were randomly varied over the full range, followed by ten steps of energy minimization in vacuum. Each structure with energy less than $500 \mathrm{~kJ}$ above the lowest energy was kept, allowing the rapid generation of 5000 quasi-random structures out of 10,000 iterations. The second method varied only the glycosidic dihedral angles and the NAc group using 500 steps of energy minimization and the GB/SA solvent model simulation of water. ${ }^{29}$ All the structures with energy below a threshold of $50 \mathrm{~kJ}(12 \mathrm{kcal})$ were kept, generating over 2000 low-energy structures for each oligosaccharide. To assay the agreement of each model with the ${ }^{1} \mathrm{D}_{\mathrm{CH}}$ data, the models were then analyzed by criteria based on relative energies and $\chi$ minimum values. Because of the limited number of significant degrees of freedom in these systems, it was found in the results to be discussed below that this lower number of structures was adequate for the dipolar coupling analysis as in a previous study of Lewis trisaccharides. ${ }^{20}$ For all the model structures, an estimated experimental error of $0.5 \mathrm{~Hz}$ was considered for the calculations with the orienting method.

To represent the conformational space of each linkage in the molecules, $\phi / \psi$ dipolar maps were generated by constructing a matrix of $360^{\circ} \times 360^{\circ}$ in steps of $5^{\circ}$.

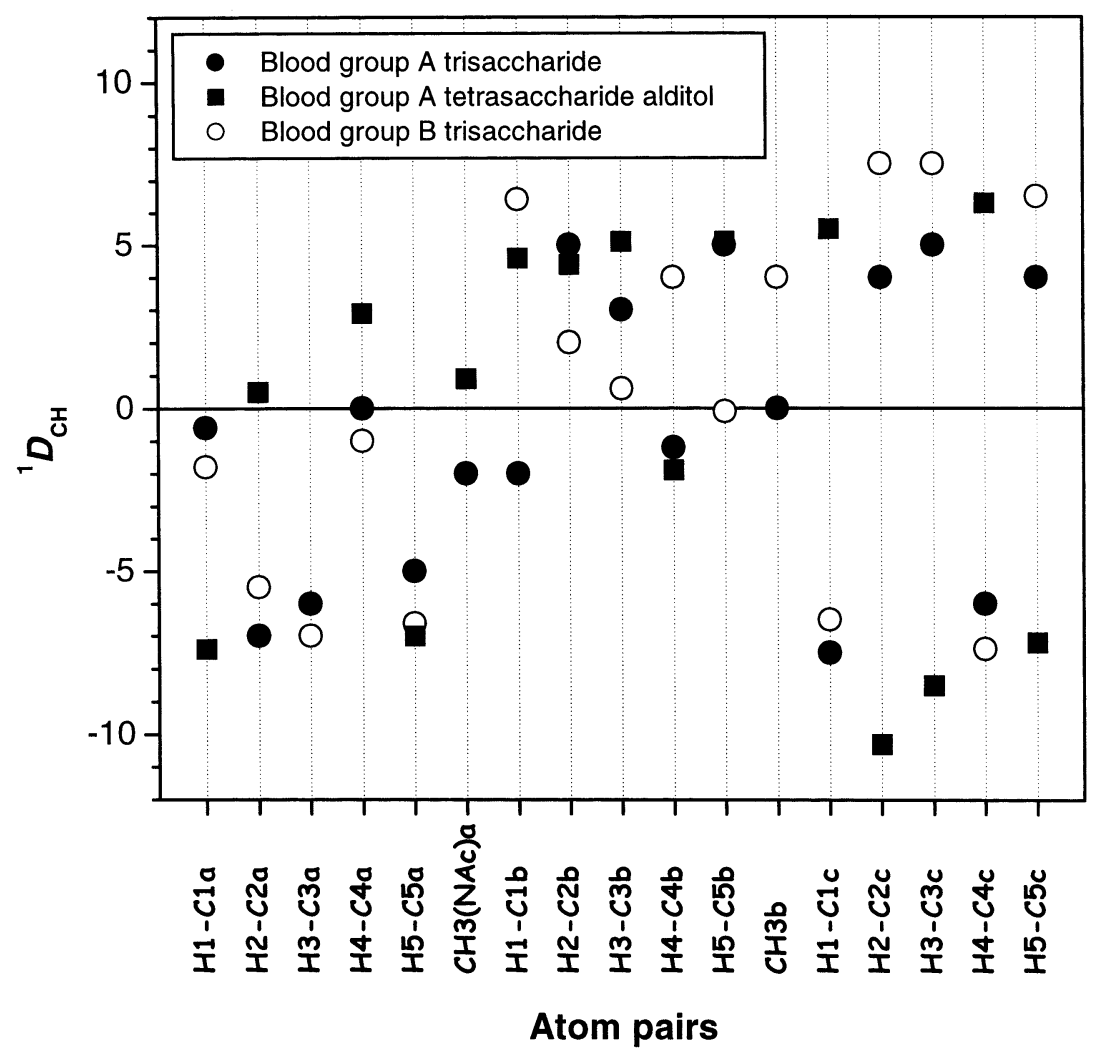

Fig. 2. Comparison of the experimental residual dipolar couplings among the compounds in Scheme 1 (a, b and c according with the scheme). 
$\chi$ (orienting)
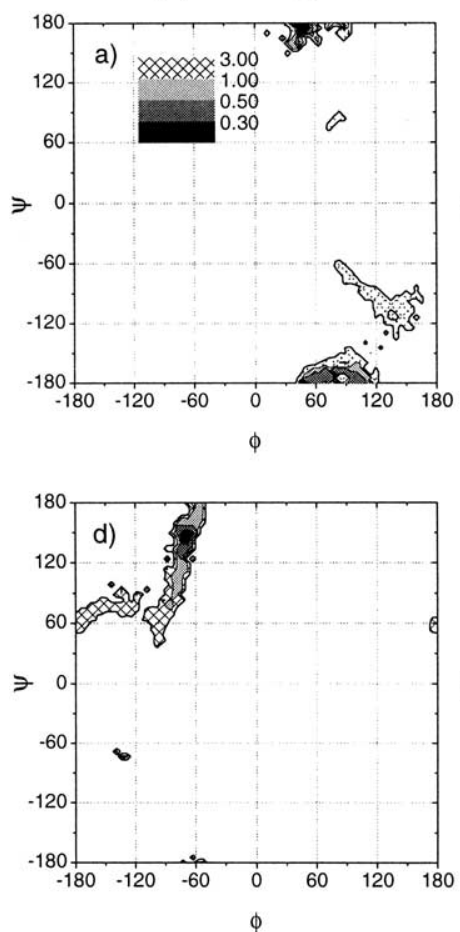

$\chi$ (TRAMITE)
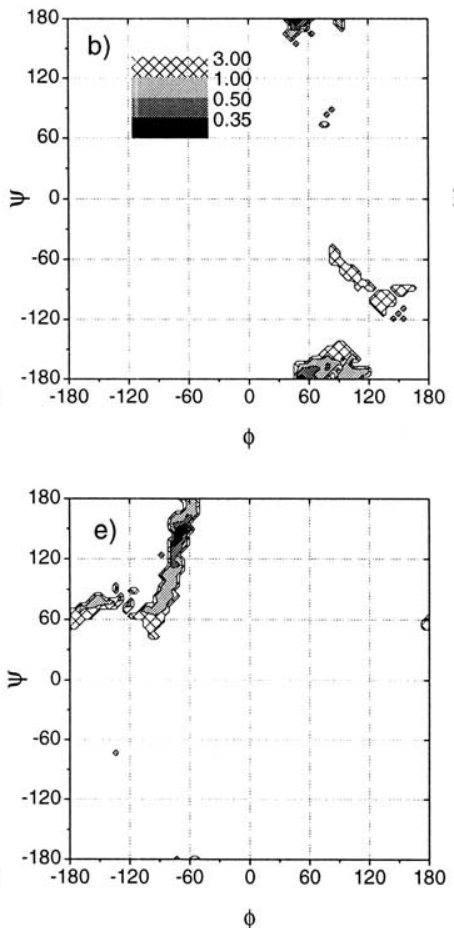

E relative $[\mathrm{kJ}]$
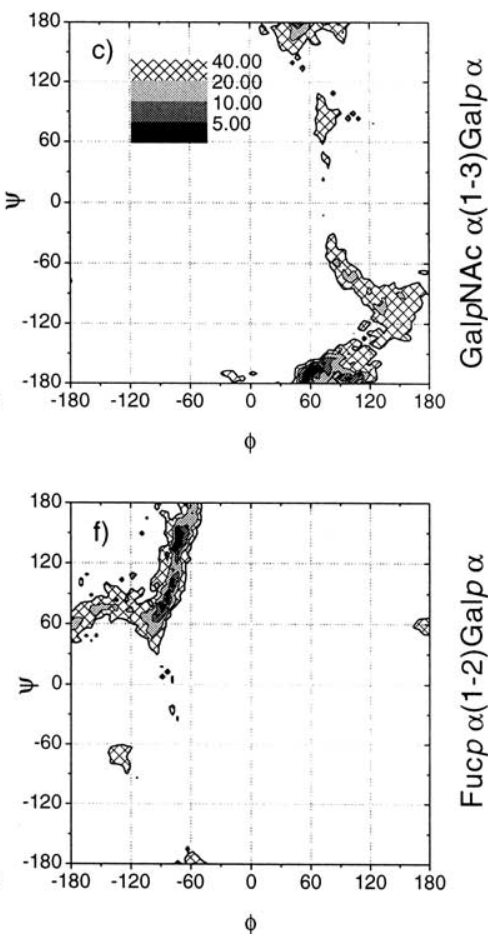

Fig. 3. Dipolar shaded maps for blood group A. (a), (b) and (c) maps for linkage Gal $p$ NAc $\alpha(1 \rightarrow 3)$ Gal $p \alpha$ shaded by $\chi$ from orienting the molecules, $\chi$ from the tensors of inertia and relative energy, respectively. (d), (e) and (f) maps for linkage Fucp $\alpha(1 \rightarrow 2) \mathrm{Gal} p \alpha$ shaded as above.

$\chi$ (orienting)
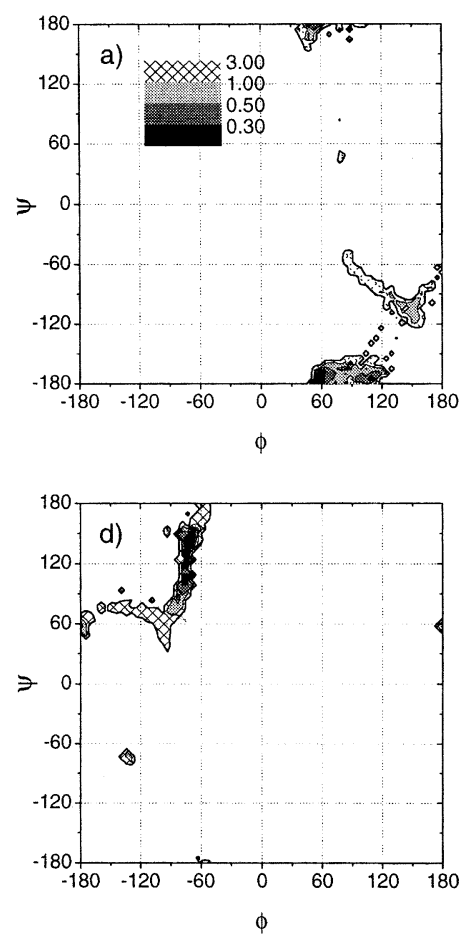

$\chi$ (TRAMITE)
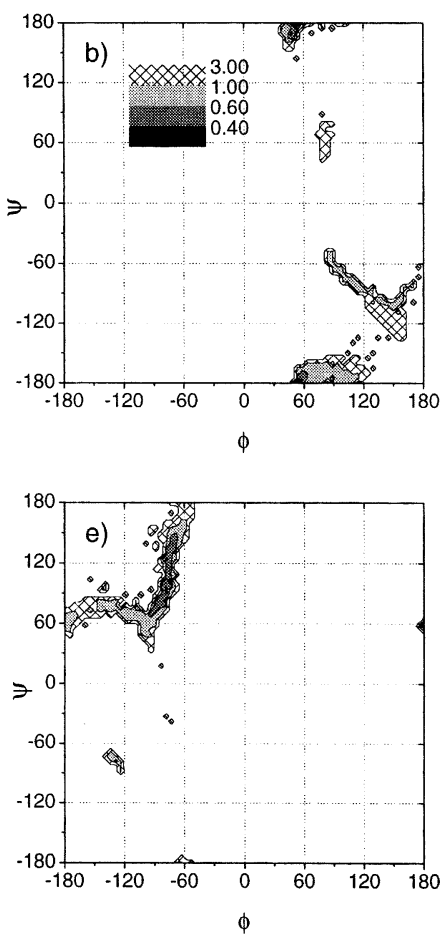

E relative $[\mathrm{kJ}]$
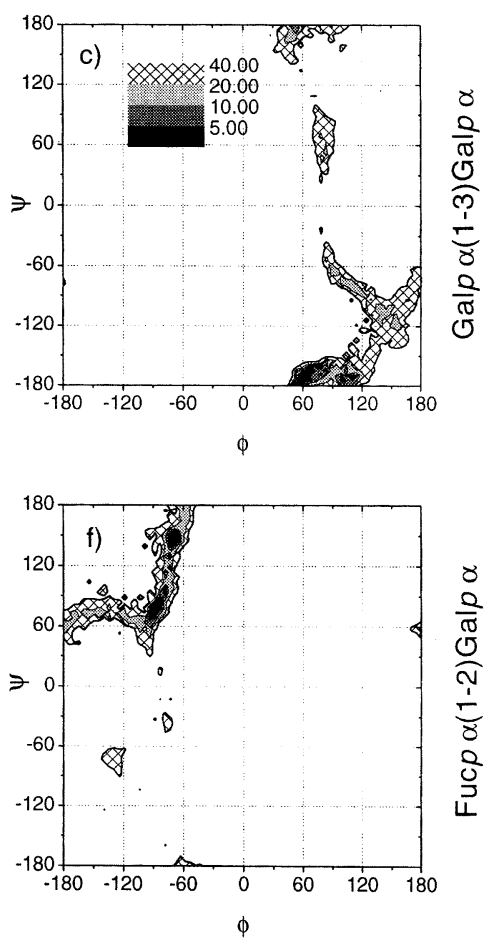

Fig. 4. Dipolar shaded maps for blood group B. (a), (b) and (c) maps for linkage Gal $p \alpha(1 \rightarrow 3)$ Gal $p \alpha$ shaded by $\chi$ from orienting the molecules, $\chi$ from the tensors of inertia and relative energy, respectively. (d), (e) and (f) maps for linkage Fucp $\alpha(1 \rightarrow 2) \mathrm{Gal} p \alpha$ shaded as above. 
To each grid point, the lowest value of either energy or $\chi$ among the models with dihedral values within the range represented by the grid point was assigned and then plotted in shaded maps. The 'random' model structures obtained by the first MC simulation resulted in very high values of $\chi$ and allow us to discard the high energy regions from the analysis (results not shown). The results obtained for the trisaccharides from the second MC simulation are shown in Figs. 3 and 4. The energy calculation shows a quite similar pattern for both the $\mathrm{A}$ and $\mathrm{B}$ trisaccharides with two conformational families having dihedral $\phi / \psi$ values for the glycosidic linkage of Fuc $\alpha(1 \rightarrow 2) \mathrm{Gal}$ of $\sim(-70 / 145)$ and $\sim(-90 / 75)$ for families I and II, respectively. There is a broader minimum for the $\alpha$-GalNAc and $\alpha$-Gal linkages that is not strongly correlated with the conformation of the fucose linkage (Fig. 3(c, f)). The results of these energy calculations are similar to those reported by Imberty et al. ${ }^{5}$

The dipolar coupling data for the blood group A trisaccharide agree only with the conformational model of family I (Fig. 3). The models giving the best agreement with experiment are very similar regardless of whether the full optimization of the orientation tensor is used (Fig. 3(a, d)) or whether the orientation tensor is calculated from the moment of intertia tensor of the model (Fig. 3(b, e)). The glycosidic dihedral angles of the best models are given in Table 3. Likewise, the dipolar coupling data for the blood group A tetrasaccharide alditol agree only with a single conformer in family I (Fig. 5). The dihedral angles for the best conformer are very similar to those for the blood group A trisaccharide when the full optimization of the orientation tensor is used (Table 3). When the TRAMITE algorithm is used to calculate the orientation tensor from the moment of intertia of the model, the fitting is not as good since the entire tetrasaccharide, including the alditol, must be used in this case. The effect of the alditol, which may well be rather flexible, does not enter into the calculation of the optimal orientation tensor since no dipolar coupling data for the alditol residue were used in that calculation.

Although the calculation of the dipolar coupling data with the TRAMITE method for the blood group B trisaccharide does not provide a clear distinction between the two conformational families, the plots of the data in which the full calculation of the orientation tensor is used are more consistent with conformations

Table 3

Glycosidic dihedral angles for the best model structures by the different methods compared by $\chi$ values obtained from orienting the respective models

\begin{tabular}{|c|c|c|c|c|c|}
\hline Molecule & Model with & Dihedral & $\mathrm{a} \rightarrow \mathrm{c}$ & $\mathrm{b} \rightarrow \mathrm{c}$ & $\chi$ (oriented) \\
\hline \multirow[t]{8}{*}{ Blood group A trisaccharide } & $E_{\min }$ & $\phi$ & 112 & -71 & 0.67 \\
\hline & (Fam. I) & $\psi$ & -167 & 149 & \\
\hline & $E_{\min }$ & $\phi$ & 69 & -79 & 1.1 \\
\hline & (Fam. II) & $\psi$ & -160 & 89 & \\
\hline & $\chi_{\min }$ & $\phi$ & 51 & -74 & 0.26 \\
\hline & (TRAMITE) & $\psi$ & -179 & 137 & \\
\hline & $\chi_{\min }$ & $\phi$ & 49 & -68 & 0.15 \\
\hline & (oriented) & $\psi$ & 179 & 146 & \\
\hline \multirow[t]{8}{*}{ Blood group B trisaccharide } & $E_{\min }$ & $\phi$ & 58 & -69 & 0.25 \\
\hline & (Fam. I) & $\psi$ & -172 & 143 & \\
\hline & $E_{\min }$ & $\phi$ & 68 & -81 & 1.00 \\
\hline & (Fam. II) & $\psi$ & -159 & 84 & \\
\hline & $\chi_{\min }$ & $\phi$ & 53 & -73 & 0.25 \\
\hline & (TRAMITE) & $\psi$ & -180 & 104 & \\
\hline & $\chi_{\min }$ & $\phi$ & 59 & -66 & 0.00 \\
\hline & (oriented) & $\psi$ & -170 & 146 & \\
\hline \multirow[t]{8}{*}{ Blood group A alditose } & $E_{\min }$ & $\phi$ & 58 & -66 & 0.49 \\
\hline & (Fam. I) & $\psi$ & -169 & 150 & \\
\hline & $E_{\min }$ & $\phi$ & 108 & -120 & 1.41 \\
\hline & (Fam. II) & $\psi$ & -168 & 83 & \\
\hline & $\chi_{\min }$ & $\phi$ & 118 & -68 & 1.58 \\
\hline & (TRAMITE) & $\psi$ & -165 & 153 & \\
\hline & $\chi_{\min }$ & $\phi$ & 60 & -66 & 0.26 \\
\hline & (oriented) & $\psi$ & -169 & 147 & \\
\hline
\end{tabular}

The linkage are indicated according to Scheme 1. 
$\chi$ (orienting)

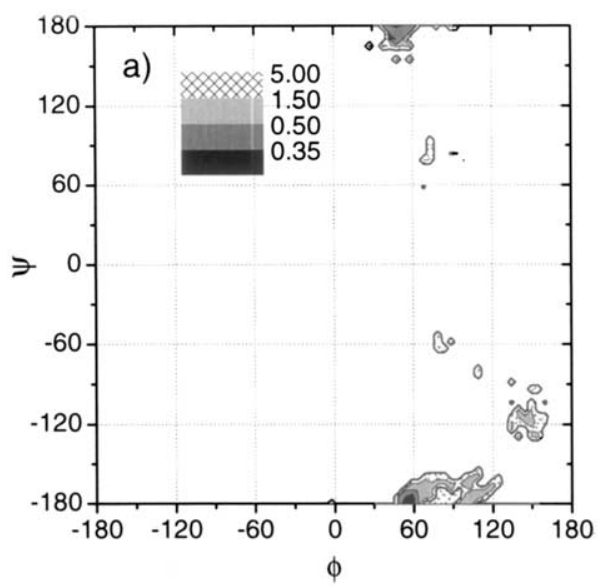

$\frac{0}{\pi}$
0
$\frac{0}{9}$
$\frac{1}{8}$
0
$\frac{1}{2}$
$\frac{0}{\pi}$
0
0

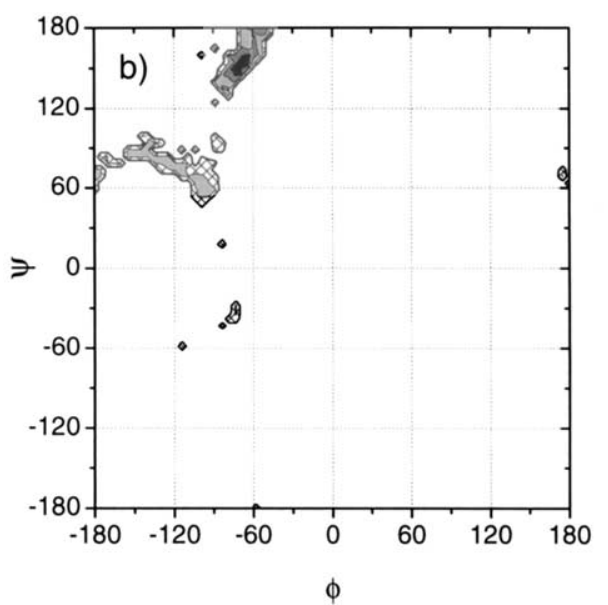

Fig. 5. Dipolar maps for blood group A $\beta(1 \rightarrow 3)$ GalNAc-ol. (a) Map for linkage $\operatorname{Gal} p \mathrm{NAc} \alpha(1 \rightarrow 3) \mathrm{Gal} p \beta$ shaded by $\chi$ from orienting the molecules. (b) Map for linkage Fuc $p \alpha(1 \rightarrow$ 2)Gal $p \beta$ shaded as above.

of family I, similar to that found for the blood group A epitope. Also, the reported long-range couplings ${ }^{3} J_{\mathrm{CH}}$ on blood group B are consistent only with the family I. ${ }^{7}$

Additional calculations were done with the TRAMITE method to evaluate the possibility of flexible conformations for the blood group epitopes. In these calculations, a series of ensembles composed of conformers selected in order of increasing energy and having $\chi$ values below 3,4 , and 5 were used to predict the ${ }^{1} D_{\mathrm{CH}}$ values. None of these ensembles gave any significant improvement in the agreement with the experimental values for either the blood group A or B trisaccharide.

\section{Conclusions}

The data suggest that single conformers adequately describe blood group A and B epitopes and that they are very similar. There are significant differences in the dipolar coupling data for blood group A and B trisaccharides that can be explained by differences in their orientation in the liquid crystal medium. The different orientations must arise from the presence of the amide function of the GalNAc residue in blood group A. Likewise, the differences in the binding of these epitopes to receptor proteins and antibodies must result from the amide group rather than from any difference in conformation.

The two families obtained in the Monte-Carlo simulations for blood group A trisaccharide found in this study were previously predicted by molecular modeling calculations in vacuum to occur in a ratio $7 / 93 .{ }^{5}$ Later $\mathrm{X}$-ray and NMR studies, based on NOE experiments, on the free compound and in the binding site of a lectin, found that the conformation bound in the active site was in family I, leading the authors to propose that an equilibrium between the two families occurs in solution with one of the conformations selected upon binding the lectin. ${ }^{6}$ The results reported here fail to support this hypothesis because a flexible solution conformation should be reflected in dipolar maps having minimum values of $\chi$ intermediate between the minimum energy conformers. Also an improvement would be expected for the predictions using ensemble averages of the moment of inertia in TRAMITE ${ }^{24}$. Our calculations using the GB/SA solvent model gave us an increased ratio between both families of 34/66 respect the previous $7 / 93$ ratio, suggesting that a more plausible explanation for the multiple energy minima is the failure of the computational models for these compounds without

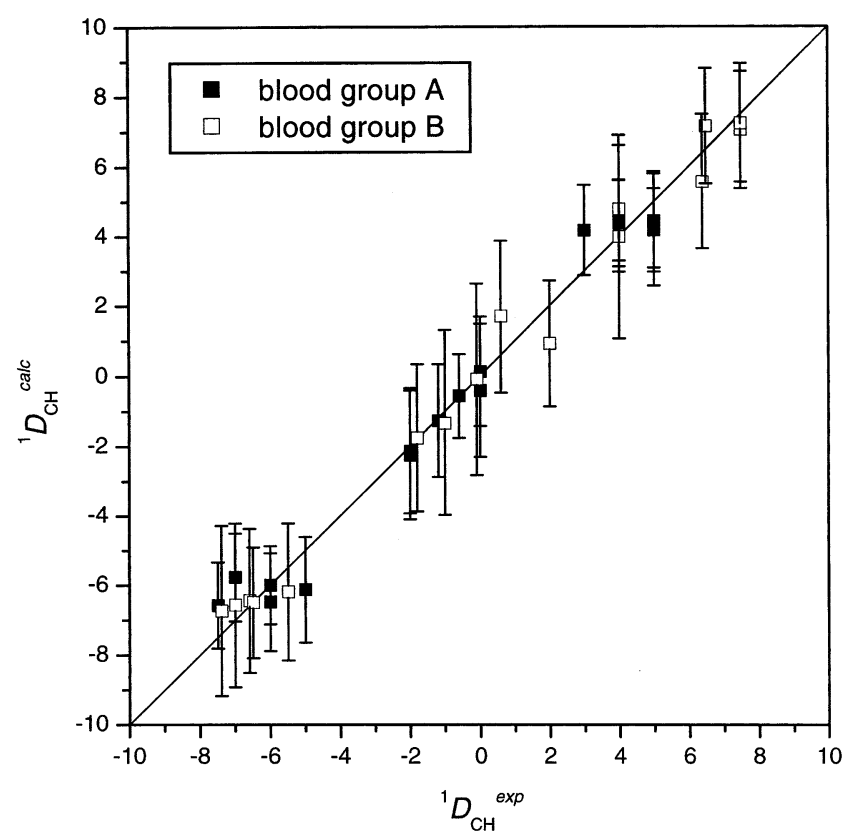

Fig. 6. Experimental versus calculated residual dipolar couplings, the bars indicating the range of calculated values obtained from the trisaccharide models whose $\chi$ value from orienting the structures was lower than 0.3 . 


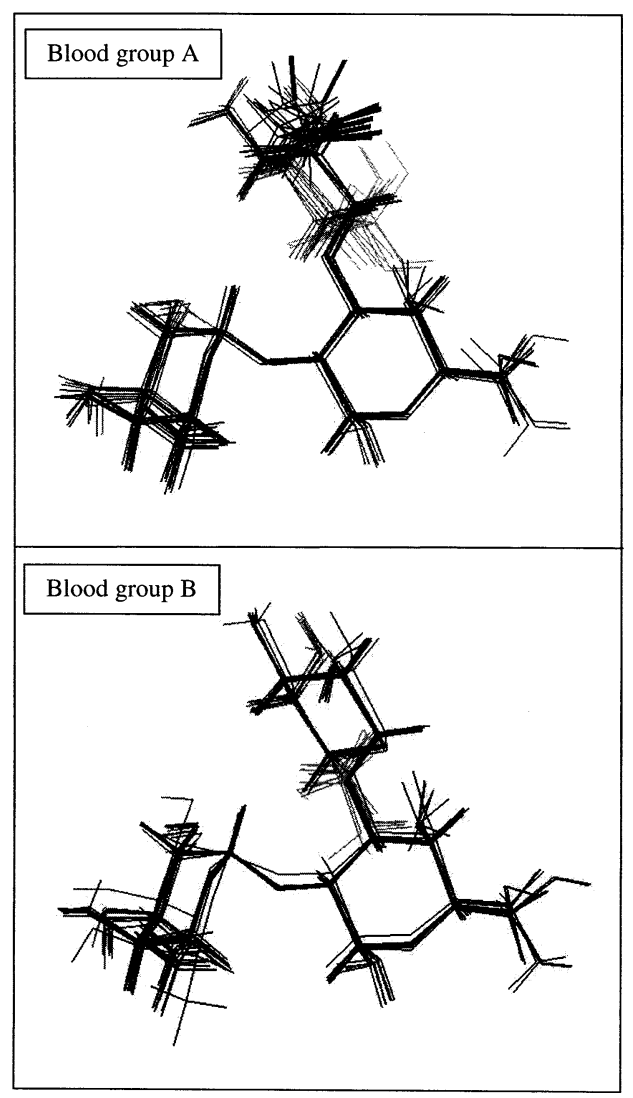

Fig. 7. The ten model structures of blood groups A and B trisaccharides that best reproduce the experimental residual dipolar coupling values.

explicit water molecules to reproduce accurately the solution conformations.

Fig. 6 shows the experimental versus calculated residual dipolar couplings for the trisaccharides with the bars indicating the range of predicted values obtained for those model structures with $\chi$ values lower than 0.3 from orienting the rigid models, 32 models for group A and 112 for group B. The range indicated in Fig. 6, about $\pm 2 \mathrm{~Hz}$, is considerably beyond our estimate of the experimental error, suggesting that many fewer models are needed to represent the conformations. A more realistic estimate of the number of models is approximately 10 . The 10 models with lowest $\chi$ values for each trisaccharide are plotted in Fig. 7. These models are conformationally very similar and agree very well with the conformations reported for blood group B by Otter et al. $^{7}$ and with the model initially proposed for solution by Bush et al. ${ }^{2}$ and obtained later by Casset et al. complexed with a lectin ${ }^{6}$ specific for blood group A.

\section{Acknowledgements}

We thank Dr Thomas Gerken of Case-Western Re- serve University for the sample of blood group A tetrasaccharide alditol. This research was supported by NIH Grant GM-57211.

\section{References}

1. Lemieux R. U.; Bock K.; Delbaere L. T. J.; Koto S.; Rao V. S. R. Can. J. Chem. 1980, 58, 631-653.

2. Bush C. A.; Yan Z. Y.; Rao N. B. N. J. Am. Chem. Soc. 1986, 108, 6168-6173.

3. Yan Z. Y.; Bush C. A. Biopolymers 1990, 29, 799811.

4. Cagas P.; Bush C. A. Biopolymers 1990, 30, 1123 1138.

5. Imberty A.; Mikros E.; Koca J.; Mollicone R.; Oriol R.; Pérez S. Glycoconjugate J. 1995, 12, 331-349.

6. Casset F.; Peters T.; Etzler M.; Korchagina E.; Nifant'ev N.; Pérez S.; Imberty A. Eur. J. Biochem. 1996, 239, $710-719$.

7. Otter A.; Lemieux R. U.; Ball R. G.; Venot A. P.; Hindsgaul O.; Bundle D. R. Eur. J. Biochem. 1999, 259, 295-303.

8. Tjandra N.; Bax A. Science 1997, 278, 1111-1114.

9. Miller K. E.; Mukhopadhyay C.; Cagas P.; Bush C. A. Biochemistry 1992, 31, 6703-6709.

10. $\mathrm{Xu} \mathrm{Q.;} \mathrm{Bush} \mathrm{C.} \mathrm{A.} \mathrm{Biochemistry} \mathrm{1996,} \mathrm{35,} \mathrm{14521-}$ 14529.

11. Poveda A.; Jiménez-Barbero J. Chem. Soc. Rev. 1998, 27, 133-143.

12. Jiménez-Barbero J.; Asensio J. L.; Cañada F. J.; Poveda A. Curr. Opin. Struct. Biol. 1999, 9, 549-555.

13. Imberty A. Curr. Opin. Struct. Biol. 1997, 7, 617623.

14. Martín-Pastor M.; Bush C. A. Biochemistry 2000, 39, 4674-4683.

15. Martín-Pastor M.; Bush C. A. Carbohydr. Res. 2000, 323, 145-147.

16. Martín-Pastor M.; Bush C. A. J. Biomol. NMR 2001, 19, 125-139.

17. Kiddle G. R.; Homans S. W. FEBS Lett. 1998, 436, $128-130$.

18. Rundlëf T.; Landersjö C.; Lycknert K.; Maliniak A.; Widmalm G. Magn. Reson. Chem. 1998, 36, 773-776.

19. Bolon P. J.; Al-Hashimi H. M.; Prestegard J. H. J. Mol. Biol. 1999, 293, 107-115.

20. Azurmendi H. F.; Martín-Pastor M.; Bush C. A. Biopolymers 2002, 63, 89-98.

21. Martín-Pastor M.; Bush C. A. Biochemistry 1999, 38, 8045-8055.

22. Almond A.; Bunkenborg J.; Franch T.; Gotfredsen C. H.; Duus J.Ø. J. Am. Chem. Soc. 2001, 123, 4792-4802.

23. Tian F.; Al-Hashimi H. M.; Craighead J. L.; Prestegard J. H. J. Am. Chem. Soc. 2001, 123, 485-492.

24. Azurmendi H. F.; Bush C. A. J. Am. Chem. Soc. 2002, 124, 2426-2427.

25. John B. K.; Plant D.; Hurd J. B. J. Magn. Reson., Ser. A 1992, 101, 113-117.

26. Ottiger M.; Delaglio F.; Marquardt J. L.; Tjandra N.; Bax A. J. Magn. Reson. 1998, 134, 365-369.

27. Mohamadi F.; Richards N. G. J.; Guida W. C.; Liskamp R.; Lipton M.; Caufield C.; Chang G.; Hendrickson T.; Still W. C. J. Comput. Chem. 1990, 11, 440-467.

28. Allinger N. L.; Yuh Y. H.; Lii J.-H. J. Am. Chem. Soc. 1989, 111, 8551-8566. 
29. Still W. C.; Tempczyk A.; Hawley R. C.; Hendrickson T. J. Am. Chem. Soc. 1990, 112, 6127-6129.

30. Parrill A. L.; Mamuya N.; Dolata D. P.; Gervay J. Glycoconjugate J. 1997, 14, 523-529.

31. Woods R. J. Glycoconjugate J. 1998, 15, 209216.

32. Clore G. M.; Gronenborn A. M.; Bax A. J. Magn. Reson. 1998, 133, 216-221.

33. Lipari G; Szabo A. J. Am. Chem. Soc. 1982, 104, 45464559.
34. Tjandra N.; Grzesiek S.; Bax A. J. Am. Chem. Soc. 1996, $118,6264-6272$.

35. Zweckstetter M.; Bax A. J. Am. Chem. Soc. 2000, 122, 3791-3792.

36. Almond A.; Bunkenborg J.; Franch T.; Gotfredsen C. H.; Duus J.Ø. J. Am. Chem. Soc. 2001, 123, 4792-4802.

37. Strecker G.; Wieruszesky J. M.; Michalsky J. C.; Montreuil J. Glycoconjugate J. 1989, 6, 271-284.

38. Dua V. K.; Rao B. N. N.; Wu S. S.; Dube V. E.; Bush C. A. J. Biol. Chem. 1986, 261, 1599-1608. 